POS $\quad$ PROCEEDINGS

\title{
Charm Quark Mass with Calibrated Uncertainty
}

\author{
Jens Erler*十 \\ Departamento de Física Teórica, Instituto de Física \\ Universidad Nacional Autónoma de México, Apartado Postal 20-364, CDMX 01000, México \\ E-mail: erlerefisica.unam.mx
}

\section{Pere Masjuan}

Grup de Física Teòrica, Departament de Física, Universitat Autònoma de Barcelona,

and Institut de Fisica d'Altes Energies, The Barcelona Institute of Science and Technology,

Campus UAB, E-08193 Bellaterra (Barcelona), Spain

E-mail: mas juaneifae.es

\section{Hubert Spiesberger}

PRISMA Cluster of Excellence, Institut für Physik

Johannes Gutenberg-Universität, 55099 Mainz, Germany

E-mail: spiesbereuni-mainz.de

\begin{abstract}
We determine the charm quark mass $m_{c}\left(m_{c}\right)$ from QCD sum rules of moments of the vector current correlator calculated in perturbative QCD. Only experimental data for the charm resonances below the continuum threshold are needed in our approach, while the continuum contribution is determined by requiring self-consistency between various sum rules, including the one for the zeroth moment. Existing data from the continuum region can then be used to bound the theoretical error. Our result is $m_{c}\left(m_{c}\right)=1272 \pm 8 \mathrm{MeV}$ for $\alpha_{s}\left(M_{Z}\right)=0.1182$. Special attention is given to the question how to quantify and justify the uncertainty.
\end{abstract}

The 39th International Conference on High Energy Physics (ICHEP2018)

4-11 July, 2018

Seoul, Korea

\footnotetext{
* Speaker.

${ }^{\dagger}$ On sabbatical leave at the PRISMA Cluster of Excellence in Mainz, Germany.
} 


\section{Method and result}

We report on work [1] on the determination of the charm quark mass, $m_{c}$. Let us recall why precise values of $m_{c}$ are needed. Besides being a fundamental input parameter defining the Standard Model (SM), it enters many QCD and electroweak processes. For example, it is needed for the renormalisation group running of the fine structure constant from the Thomson limit to the $Z$ pole [2]. Incidentally, the physics involved in this running is the basis of what we will refer to as the $0^{\text {th }}$ moment of the sum rule later on. Conversely, if one wants to run the weak mixing angle from the $Z$ pole down to low energies one can do so in perturbation theory as well, but one needs an input value for $m_{c}$ [3]. Finally, $m_{c}$ enters the SM prediction of the anomalous magnetic moment of the muon when the heavy quark contribution is evaluated perturbatively [4]. The most important future application will be the test of the mass versus Yukawa coupling relation in the single Higgs $\mathrm{SM}$, because at future lepton colliders it will be possible to measure the charm Yukawa coupling very precisely. If one - within the $\mathrm{SM}$ - converts the projected precision to a mass measurement this would correspond to an error of $\approx 8 \mathrm{MeV}$, which should be the benchmark of what one wants to achieve at least regarding the precision in $m_{c}$. One can also determine $m_{c}$ in lattice simulations, but it is prudent to inquire for a second opinion derived from an independent first principles approach.

This approach is provided by the relativistic QCD sum rule formalism describing low moments of the charm vector-current correlator $\Pi_{c}$, where the master equation is given by

$$
12 \pi^{2} \frac{\Pi_{c}(0)-\Pi_{c}(-t)}{t}=\int_{4 \hat{m}_{c}^{2}}^{\infty} \frac{\mathrm{d} s}{s} \frac{R_{c}(s)}{s+t} .
$$

The right-hand side is basically an integral over electromagnetic charm pair production (normalized to the muonic cross-section) with certain weights. $\Pi_{c}$ has been calculated in perturbative QCD to $\mathscr{O}\left(\alpha_{s}^{3}\right)[5,6,7,8]$. In the limit $t \rightarrow 0$, the left-hand side of Eq. (1.1) turns into a derivative with the right-hand side being suppressed by two powers of $s$ and providing the $1^{\text {st }}$ moment, $\mathscr{M}_{1}$. Taking further derivatives generates higher moments, $\mathscr{M}_{n}$ [9]. But one can also take the opposite limit, $t \rightarrow \infty$, which corresponds to the $0^{\text {th }}$ moment sum rule, $\mathscr{M}_{0}$ [4], mentioned already before. As it stands, $\mathscr{M}_{0}$ would be divergent and needs regularization, but this can be done e.g., by subtracting the asymptotic perturbative expansion, $R_{c}(s) \equiv 4 / 3 \lambda_{1}(s)$ at $m_{c}=0$, on both sides.

$\mathscr{M}_{0}$ is one of the ingredients where our analysis differs from others. Another special feature of our approach is that the only experimental input are the electronic widths of the $J / \psi$ and the $\psi(2 S)$ narrow vector resonances. The continuum contribution will be constrained by requiring selfconsistency between various sum rules. This is where $\mathscr{M}_{0}$ comes in handy because it experiences stronger sensitivity to the continuum compared to higher moments, and at the same time a milder sensitivity to $m_{c}$, so that it provides a useful handle. This means that quark-hadron duality is needed only in the finite region between the $\psi(2 S)$ and the onset of open charm production. By contrast, in previous analyses one performs the integral in Eq. (1.1) up to some value of $s$ after which no more data are available and where one needs to switch to perturbative QCD. This involves changing from hadron to quark degrees of freedom at this particular point which is not rigorously justified. Finally, in our approach it is also possible to estimate correlated errors across various moments. Our result for the $\overline{\mathrm{MS}}$ charm quark mass is

$$
\hat{m}_{c}\left(\hat{m}_{c}\right)=1272 \pm 8+2616\left[\hat{\alpha}_{s}\left(M_{Z}\right)-0.1182\right] \mathrm{MeV}
$$


where the theoretical and experimental uncertainties add up to $8 \mathrm{MeV}$, coinciding with the benchmark target mentioned earlier. In addition, we explicitly display the parametric dependence on the strong coupling constant, $\hat{\alpha}_{s}\left(M_{Z}\right)$. For this particular result - the main result of our work - we used $\mathscr{M}_{0}$ in tandem with $\mathscr{M}_{2}$, where we assumed the error component associated with the truncation of perturbative QCD to be uncorrelated between the two (we expect this error contribution to be stronger correlated among the higher moments). The central value is in very good agreement with other recent sum rule determinations but there is less agreement regarding the theory dominated uncertainty, so we wish to go into a few more details about it.

\section{Discussion}

Figure 1 shows the total uncertainty and the error breakdown as a function of the order in $\hat{\alpha}_{s}$. The experimental input error from the electronic partial widths is shown in red. For the truncation error (in green) we used a somewhat more conservative estimate than simply taking the last available term. As for the gluon condensate, we take the entire estimated contribution as the uncertainty (in orange). We also show the parametric uncertainty (in purple) from $\hat{\alpha}_{s}\left(M_{Z}\right)=0.1182 \pm 0.0016$. Finally, we want to allow for the possibilities of larger-than-expected duality violations (while the assumption of quark-hadron duality in a finite region is much weaker than local duality, it still lacks complete rigour) or high-order terms in the operator product expansion (OPE), given that the charm quark mass is dangerously close to the hadronic scale. Therefore, we use $e^{+} e^{-} \rightarrow$ hadrons electro-production data as our control of the method, and compare the constraints on the continuum region from internal consistency by simultaneously considering different sum rules on the one hand with actual measurements on the other. We add the difference between the two constraints and the uncertainty in the experimental continuum data itself in quadrature (in cyan).

Figure 2 reveals good agreement within the stated errors with other sum rule determinations of $\hat{m}_{c}\left(\hat{m}_{c}\right)$ using $\mathscr{M}_{1}[10,11], \mathscr{M}_{2}$ [12], and higher moments [13], as well as with $1 S$-charmonium spectroscopy [14]. Our result also agrees very well with those from lattice simulations based on the pseudo-scalar current [15], domain-wall fermions [16], and staggered quarks [17].

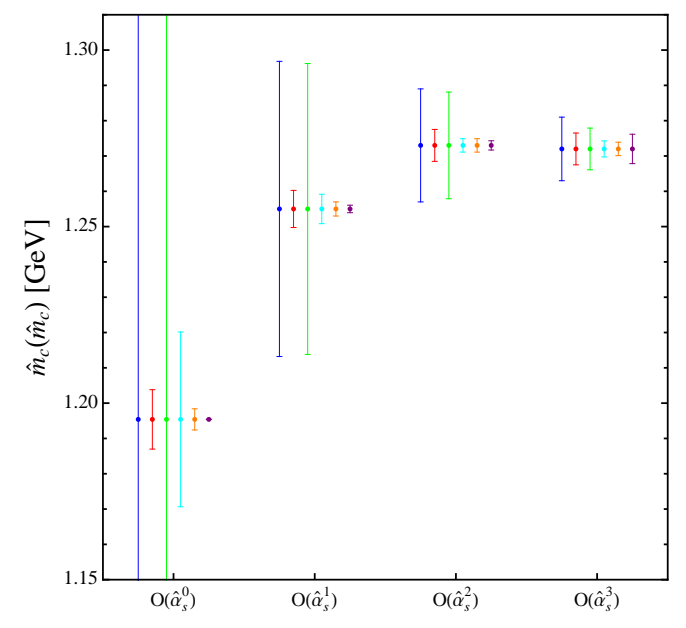

Figure 1: Error budget for $\hat{m}_{c}\left(\hat{m}_{c}\right)$ when determined from the pair of moments $\left(\mathscr{M}_{0}, \mathscr{M}_{2}\right)$ at different orders. 


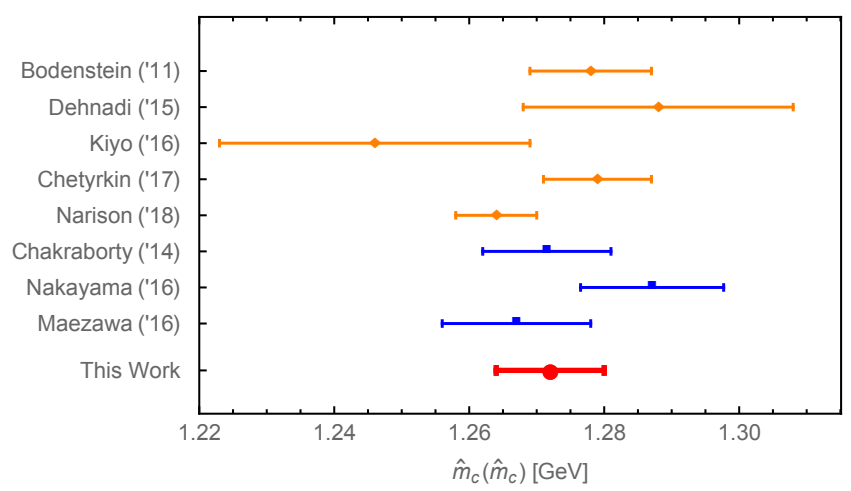

Figure 2: Recent charm quark mass determinations (see text for details and references).

\section{Acknowledgments}

This work has been supported by DFG through the Collaborative Research Center "The LowEnergy Frontier of the Standard Model" (SFB 1044), by CONACyT (Mexico) project 252167-F and the German-Mexican research collaboration grant SP 778/4-1 (DFG) and 278017 (CONACyT). JE acknowledges support from the Mainz cluster of excellence PRISMA.

\section{References}

[1] J. Erler, P. Masjuan and H. Spiesberger, Eur. Phys. J. C 77 (2017) 99 [arXiv: 1610.08531 ].

[2] J. Erler, Phys. Rev. D 59 (1999) 054008 [hep-ph/ 9803453$].$

[3] J. Erler and M. J. Ramsey-Musolf, Phys. Rev. D 72 (2005) 073003 [lhep-ph / 0409169 ].

[4] J. Erler and M. Luo, Phys. Lett. B 558 (2003) 125 [hep-ph/ 0207114$].$

[5] K. G. Chetyrkin, J. H. Kühn and C. Sturm, Eur. Phys. J. C 48 (2006) 107 [hep-ph/ 0604234 ].

[6] R. Boughezal, M. Czakon and T. Schutzmeier, Phys. Rev. D 74 (2006) 074006 [hep-ph / 0605023 ].

[7] B. A. Kniehl and A. V. Kotikov, Phys. Lett. B 642 (2006) 68 [hep-ph/ 0607201$].$

[8] A. Maier et al., Nucl. Phys. B 824 (2010) 1 [arXiv:0907.2117].

[9] V. A. Novikov et al., Phys. Rept. 41 (1978) 1.

[10] B. Dehnadi, A. H. Hoang and V. Mateu, JHEP 1508 (2015) 155 [arXiv: 1504.07638 ].

[11] K. G. Chetyrkin et al., Phys. Rev. D 96 (2017) 116007 [arXiv:1710. 04249 ].

[12] S. Bodenstein et al., Phys. Rev. D 83 (2011) 074014 [arXiv: 1102 . 3835].

[13] S. Narison, Phys. Lett. B 784 (2018) 261 [arXiv: 1808 . 01969].

[14] Y. Kiyo, G. Mishima and Y. Sumino, Phys. Lett. B 752 (2016) 122 [arXiv: 1510 . 07072].

[15] B. Chakraborty et al., Phys. Rev. D 91 (2015) 054508 [arXiv: 1408 . 4169].

[16] K. Nakayama, B. Fahy and S. Hashimoto, Phys. Rev. D 94 (2016) 054507 [arXiv: 1606.01002 ].

[17] Y. Maezawa and P. Petreczky, Phys. Rev. D 94 (2016) 034507 [arXiv:1606. 08798]. 